Entropy 2003, 5, 482-495

\title{
Entropy
}

ISSN 1099-4300

www.mdpi.org/entropy/

\section{Analytical Entropy Analysis of Recuperative Heat Exchangers}

\author{
Antun Galović*, Zdravko Virag*, Marija Živić** \\ *University of Zagreb, Faculty of Mechanical Engineering and Naval Architecture, I. Lučića 5, \\ 10000 Zagreb, Croatia, (e-mails: antun.galovic@fsb.hr and zdravko.virag@fsb.hr) \\ **University of Osijek, Mechanical Engineering Faculty Trg I. B. Mažuranić 18, \\ 35000 Slavonski Brod, Croatia (e-mail: marija.zivic@sfsb.hr)
}

Received: 22 July 2003 / Accepted: 28 November 2003 / Published: 31 December 2003

\begin{abstract}
The analytical solutions for the temperature variation of two streams in parallel flow, counter flow and cross-flow heat exchangers and related entropy generation due to heat exchange between the streams are presented. The analysis of limiting cases for the relative entropy generation is performed, and corresponding analytical expressions are given. The obtained results may be included in a more general procedure concerning optimal heat exchanger design.
\end{abstract}

Key words: Recuperative Heat Exchangers, Relative Entropy Generation, Analytical Solution

\section{Introduction}

Many years ago Bošnjaković [1] has pointed out the necessity of introducing entropy generation into the analysis of heat process apparatus. Recuperative heat exchangers are typical examples of such apparatus. There are two sources of entropy generation in heat exchangers. One is due to heat exchange between two streams of different temperature, and the second one due to viscosity of moving fluids. The entropy generation rate due to viscosity is usually much smaller than the generation due to heat exchange, so it can be neglected. In [2,3] one can find a detailed analysis of entropy generation calculated from integral balance along streamlines and normalized by the heat capacity rate of one of the streams. Some more recent papers [4,5] deal with the entropy analysis of heat exchangers in a similar way. In this paper the three types of heat exchangers are analyzed and entropy generation rate due to heat exchange is calculated exactly, following the integration of entropy generation rate over the exchanger surface. The relative entropy generation rate is introduced and shown as a function of the same parameters for all types of heat exchangers. Analytical solutions are presented and analyzed. 


\section{Analytical Relationships for Heat Exchangers}

The analyzed heat exchangers may be considered as a dividing wall between two fluids. Any of them is characterized by the wall surface area $A_{0}$, overall heat exchange coefficient $\mathrm{k}$, heat capacity rates of the weaker and stronger stream $C_{1}$ and $C_{2}$ and inlet temperatures of two streams $T_{1}^{\prime}$ and $T_{2}^{\prime}$. Instead of these six dimensional variables it is convenient to introduce the following three non-dimensional parameters:

$\pi_{2}=\frac{k A_{0}}{C_{1}}, \pi_{3}=\frac{C_{1}}{C_{2}}$ and $\pi_{T}=\frac{T_{2}^{\prime}}{T_{1}^{\prime}}$

For the each type of the considered heat exchangers, the analytical solution for the temperature distribution in both streams exists, so it is possible to find the heat transfer rate between two streams

$$
\Phi=k \int_{A_{0}}\left(T_{1}-T_{2}\right) \mathrm{d} A
$$

and the entropy generation rate due to heat transfer. According to [6,7], this part of entropy generation rate is defined as

$$
\dot{S}_{\text {gen }}=k \int_{A_{0}} \frac{\left(T_{1}-T_{2}\right)^{2}}{T_{1} T_{2}} \mathrm{~d} A
$$

Usual dimensionless forms of the heat transfer rate and the entropy generation rate are

$$
\begin{aligned}
& \mathcal{E}=\pi_{1}=\frac{\Phi}{C_{1}\left(T_{1}^{\prime}-T_{2}^{\prime}\right)}=\frac{\Phi}{\Phi_{\max }} \\
& N_{S}=\frac{\dot{S}_{\text {gen }}}{k A_{0}}
\end{aligned}
$$

where $\varepsilon$ is heat exchanger effectiveness and $N_{S}$ the entropy generation number.

\subsection{Solution for the Parallel Flow Heat Exchanger}

The temperature distribution in the streams of a parallel flow heat exchanger (PAHE) is defined as [7]

$$
\frac{T_{1}}{T_{1}^{\prime}}=\frac{\pi_{\mathrm{T}}+\pi_{3}}{1+\pi_{3}}+\frac{1-\pi_{\mathrm{T}}}{1+\pi_{3}} \mathrm{e}^{-\pi_{2}\left(1+\pi_{3}\right) \frac{A}{A_{0}}}
$$




$$
\frac{T_{2}}{T_{1}^{\prime}}=\frac{\pi_{T}+\pi_{3}}{1+\pi_{3}}-\pi_{3} \frac{1-\pi_{T}}{1+\pi_{3}} \mathrm{e}^{-\pi_{2}\left(1+\pi_{3}\right) \frac{A}{A_{0}}}
$$

for the following boundary conditions: for $A=0, T_{1}=T_{1}^{\prime}$ and for $A=A_{0}, T_{2}=T_{2}^{\prime}$. By using Eqs. (3), (6) and (7), the expression for the entropy generation number follows

$$
\begin{aligned}
N_{\mathrm{S}}= & \frac{\left(1+\pi_{3}\right)}{2 \pi_{2} \pi_{3}} \ln \left|\frac{\pi_{3}\left(1-\pi_{\mathrm{T}}\right)^{2} \mathrm{e}^{-\left(1+\pi_{3}\right) \pi_{2}}-\left(\pi_{\mathrm{T}}+\pi_{3}\right)^{2}\left[\frac{1-\pi_{3}}{\pi_{\mathrm{T}}+\pi_{3}}\left(1-\pi_{\mathrm{T}}\right) \mathrm{e}^{-\left(1+\pi_{3}\right) \pi_{2}}+1\right]}{\pi_{3}\left(1-\pi_{\mathrm{T}}\right)^{2}-\left(\pi_{\mathrm{T}}+\pi_{3}\right)\left(1+\pi_{\mathrm{T}} \pi_{3}\right)}\right| \\
& \left.+\frac{\left(1-\pi_{3}\right)}{2 \pi_{2} \pi_{3}} \ln \mid \frac{\pi_{\mathrm{T}}+\pi_{3}-\pi_{3}\left(1-\pi_{\mathrm{T}}\right) \mathrm{e}^{-\left(1+\pi_{3}\right) \pi_{2}}}{\pi_{\mathrm{T}}\left[\pi_{T}+\pi_{3}+\left(1-\pi_{\mathrm{T}}\right) \mathrm{e}^{-\left(1+\pi_{3}\right) \pi_{2}}\right.}\right]
\end{aligned}
$$

\subsection{Counterflow heat exchanger}

The temperature distribution in the streams of a counterflow heat exchanger (COHE) is defined as [7]

$$
\begin{aligned}
& \frac{T_{1}}{T_{1}^{\prime}}=\frac{\pi_{T} \mathrm{e}^{-\left(\pi_{3}-1\right) \pi_{2}}-1+\left(1-\pi_{T}\right) \mathrm{e}^{-\pi_{2}\left(\pi_{3}-1\right) \frac{A}{A_{0}}}}{\mathrm{e}^{-\left(\pi_{3}-1\right) \pi_{2}}-\pi_{3}} \\
& \frac{T_{2}}{T_{1}^{\prime}}=\frac{\pi_{T} \mathrm{e}^{-\left(\pi_{3}-1\right) \pi_{2}}-1-\pi_{3}\left(1-\pi_{T}\right) \mathrm{e}^{-\pi_{2}\left(\pi_{3}-1\right) \frac{A}{A_{0}}}}{\mathrm{e}^{-\left(\pi_{3}-1\right) \pi_{2}}-\pi_{3}}
\end{aligned}
$$

for the following boundary conditions: for $A=0, T_{2}=T_{2}^{\prime}$ and for $A=A_{0}, T_{1}=T_{1}^{\prime}$. By using Eqs. (3), (9) and (10), the expression for the entropy generation number becomes

$$
\begin{aligned}
N_{\mathrm{S}}= & \frac{1-\pi_{3}}{2 \pi_{2} \pi_{3}} \ln \left|\frac{\pi_{3}\left(1-\pi_{\mathrm{T}}\right)^{2} \mathrm{e}^{-\left(\pi_{3}-1\right) \pi_{2}}+\left(\pi_{T} \mathrm{e}^{-\left(\pi_{3}-1\right) \pi_{2}}\right)\left(\mathrm{e}^{-\left(\pi_{3}-1\right) \pi_{2}}+\pi_{3}\left(1-\pi_{T}\right) \mathrm{e}^{-\left(\pi_{3}-1\right) \pi_{2}}-1\right)}{\pi_{3}\left(1-\pi_{T}\right)^{2}+\left(\pi_{T} \mathrm{e}^{-\left(\pi_{3}-1\right) \pi_{2}}-\pi_{3}\right)\left(1-\pi_{T}-\pi_{T}\left(\pi_{3}-\mathrm{e}^{-\left(\pi_{3}-1\right) \pi_{2}}\right)\right)}\right| \\
& -\frac{1+\pi_{3}}{2 \pi_{2} \pi_{3}} \ln \left|\frac{\left(\pi_{3} \mathrm{e}^{-\left(\pi_{3}-1\right) \pi_{2}}-\pi_{3}\right) \mathrm{e}^{\left(-\left(\pi_{3}-1\right) \pi_{2}\right)}-\pi_{3}+\pi_{T}}{A_{1}\left[\pi_{T}+\pi_{3}-\pi_{T} \mathrm{e}^{-\left(\pi_{3}-1\right) \pi_{2}}-1\right]}\right| \\
A_{1}= & \pi_{T} \mathrm{e}^{-\left(\pi_{3}-1\right) \pi_{2}}\left(1-\pi_{3}\right)-\pi_{3}\left(1-\mathrm{e}^{-\left(\pi_{3}-1\right) \pi_{2}}\right)
\end{aligned}
$$




\subsection{Cross-flow heat exchanger}

In a cross flow heat exchanger (CRHE) the streams are at an angle, so the temperatures of both streams depend on two coordinates, the non-dimensional $\mathrm{x}$ and $\mathrm{y}$. The analytical solution is then defined by the infinite series [8]

$$
\begin{aligned}
& \frac{T_{1}}{T_{1}^{\prime}}=\pi_{T}+\left(1-\pi_{T}\right)\left\{1-e^{-(x+y)}\left[x+\frac{x^{2}}{2 !}(1+y)+\ldots .+\frac{x^{n}}{n !}\left(1+y+\ldots+\frac{y^{n-1}}{(n-1) !}\right)+\ldots\right]\right\} \\
& \frac{T_{2}}{T_{1}^{\prime}}=\pi_{T}+\left(1-\pi_{T}\right)\left\{1-e^{-(x+y)}\left[1+x(1+y)+\ldots+\frac{x^{n}}{n !}\left(1+y+\ldots+\frac{y^{n}}{n !}\right)+\ldots\right]\right\}
\end{aligned}
$$

Using the above formulas it is possible to calculate the temperatures $T_{1}$ and $T_{2}$ up to a prescribed accuracy. The same applies for the numerical integration of the total heat flux between streams and the entropy generation rate [9].

\subsection{Limiting cases of the entropy number}

When one of the streams evaporates or condenses $\left(\pi_{3}=0\right)$ the entropy number is the same for all three types of heat exchangers. It follows from Eq. (8) or (11)

$$
N_{S}\left(\pi_{2}, \pi_{T}, \pi_{3}=0\right)=\frac{1}{\pi_{2}}\left[\ln \left|\mathrm{e}^{-\pi_{2}}+\pi_{T}\left(1-\mathrm{e}^{-\pi_{2}}\right)\right|\right]+\frac{1}{\pi_{2}}\left[\left(\frac{1}{\pi_{T}}-1\right)\left(1-\mathrm{e}^{-\pi_{2}}\right)\right]
$$

Eq. (11), for a counterflow heat exchanger is the indeterminate form $0 / 0$ for $\pi_{3}=1$. The limit can be found by using L'Hospital's rule

$$
N_{\mathrm{S}}\left(\pi_{2}, \pi_{T}, \pi_{3}=1\right)=\frac{1}{\pi_{2}} \ln \frac{\left(\pi_{T}+\pi_{2}\right)\left(1+\pi_{2} \pi_{T}\right)}{\left(\pi_{2}+1\right)^{2} \pi_{T}}
$$

When both streams evaporate or condense $\left(C_{1} \rightarrow \infty\right.$ and $C_{2} \rightarrow \infty ; \pi_{2}=0$ and $\left.\pi_{3}=1\right)$, the maximal entropy number is the same for all types of heat exchangers. It is defined by

$$
N_{S, \max }=\frac{\left(\pi_{T}-1\right)^{2}}{\pi_{T}}
$$




\subsection{Relative entropy generation}

If the maximum entropy number is known, the relative entropy generation can be defined as

$\varepsilon_{S}=\frac{N_{\mathrm{S}}}{N_{\mathrm{S}, \text { max }}}=\frac{\dot{S}_{\mathrm{gen}}}{\dot{S}_{\mathrm{gen}, \text { max }}}$

It is well known that the heat exchanger effectiveness depends on parameters $\pi_{2}$ and $\pi_{3}$. It is possible to express the parameter $\pi_{2}$ as a function of $\varepsilon$ and $\pi_{2}$. For the parallel flow heat exchanger the function is given in [7].

$\pi_{2}=\frac{1}{1+\pi_{3}} \ln \left|\frac{1}{1-\varepsilon\left(1+\pi_{3}\right)}\right|$

and for counterflow heat exchanger it is

$\pi_{2}=\frac{1}{1-\pi_{3}} \ln \left|\frac{1-\varepsilon \pi_{3}}{1-\varepsilon}\right|$

For $\pi_{3}=0$ this relationship is the same for the all three types of heat exchangers

$\pi_{2}\left(\varepsilon, \pi_{3}=0\right)=\ln \left|\frac{1}{1-\varepsilon}\right|$

The relative entropy generation depends on the parameters $\pi_{2}, \pi_{3}$ and $\pi_{\mathrm{T}}$, but it may be rearranged into a form

$\varepsilon_{\mathrm{S}}=f\left(\varepsilon, \pi_{3}, \pi_{T}\right)$

In the next section this relationship is presented graphically for the all three types of heat exchangers.

\section{Graphical Presentation of the Entropy Generation Rate}

\subsection{General case}

Figures $1 \mathrm{a}$ to $2 \mathrm{~b}$ show the relative entropy generation versus heat exchanger effectiveness for the parallel flow heat exchanger. Figs. $1 \mathrm{a}$ and $1 \mathrm{~b}$ correspond to $\pi_{3}=0.5$, while Figs. $2 \mathrm{a}$ and $2 \mathrm{~b}$ are for $\pi_{3}=1$. In Figs. 1a and $2 \mathrm{a}$ the parameter $\pi_{T}$ varies from 0.2 to 1 , and in Figs. $1 \mathrm{~b}$ and $2 \mathrm{~b}$ from 1 to 10. 


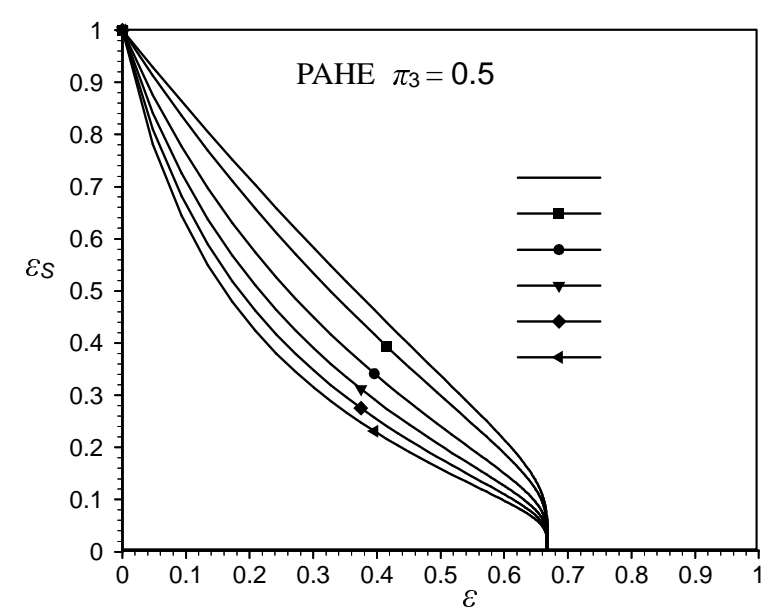

Figure 1a Relative entropy generation $\varepsilon_{S}$ as a function of the heat exchanger effectiveness $\varepsilon$ for $\pi_{3}=0.5$ and $\pi_{T}=0.2$ to 1 , for a parallel flow heat exchanger

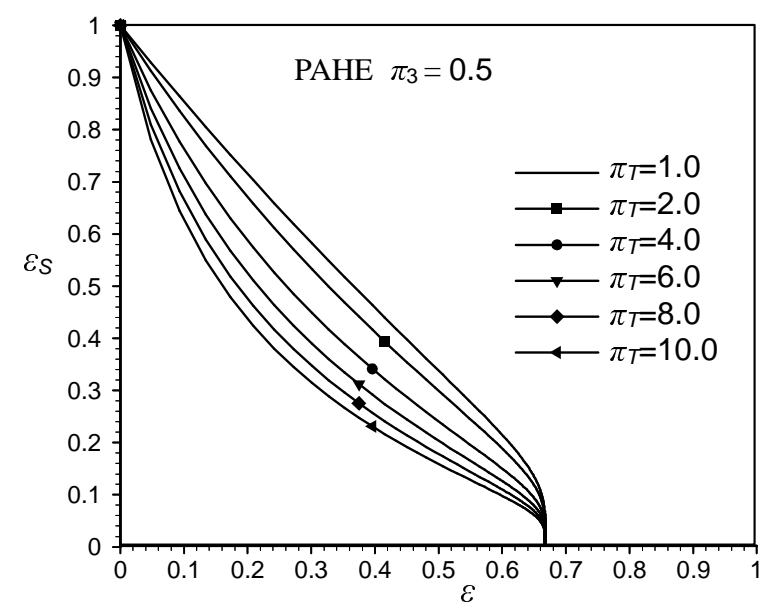

Figure 1b Relative entropy generation $\varepsilon_{S}$ as a function of the heat exchanger effectiveness $\varepsilon$ for $\pi_{3}=0.5$ and $\pi_{T}=1$ to 10 , for a parallel flow heat exchanger

Eq.(18) clearly indicates that for $\pi_{3}=0.5$ and $\pi_{2} \rightarrow \infty$ the effectiveness of a parallel flow heat exchanger tends to $2 / 3$, as it shown in Figures $1 \mathrm{a}$ and $1 \mathrm{~b}$. From these diagrams it may be seen that $\varepsilon_{\mathrm{S}}$ increases with $\pi_{T}$ increasing from 0.2 to 1 and decreases again with $\pi_{T}$ increasing 1 to 10 .

For $\pi_{3}=1$ the limiting value of the parallel flow heat exchanger effectiveness is 0.5 , as it is shown in Figs. $2 \mathrm{a}$ and $2 \mathrm{~b}$. 


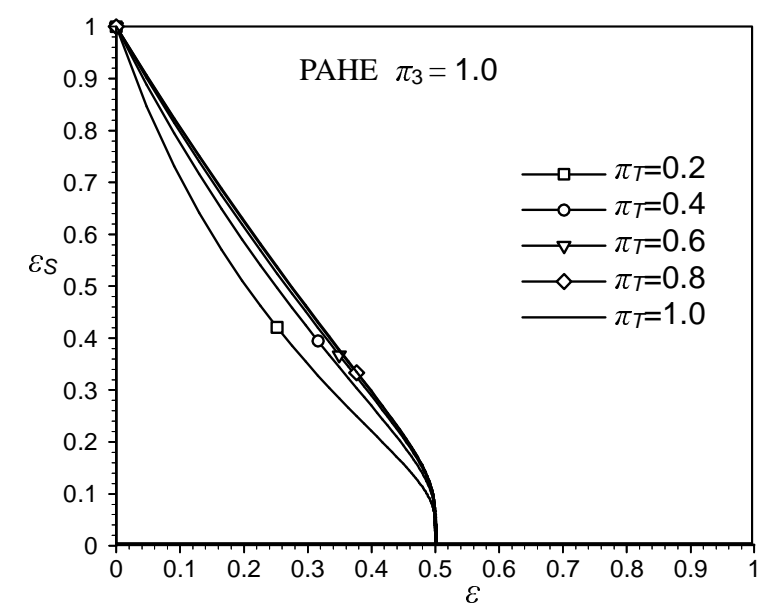

Figure 2a Relative entropy generation $\varepsilon_{S}$ as a function of the heat exchanger effectiveness $\varepsilon$ for $\pi_{3}=1.0$ and $\pi_{T}=0.2$ to 1 , for a parallel flow heat exchanger

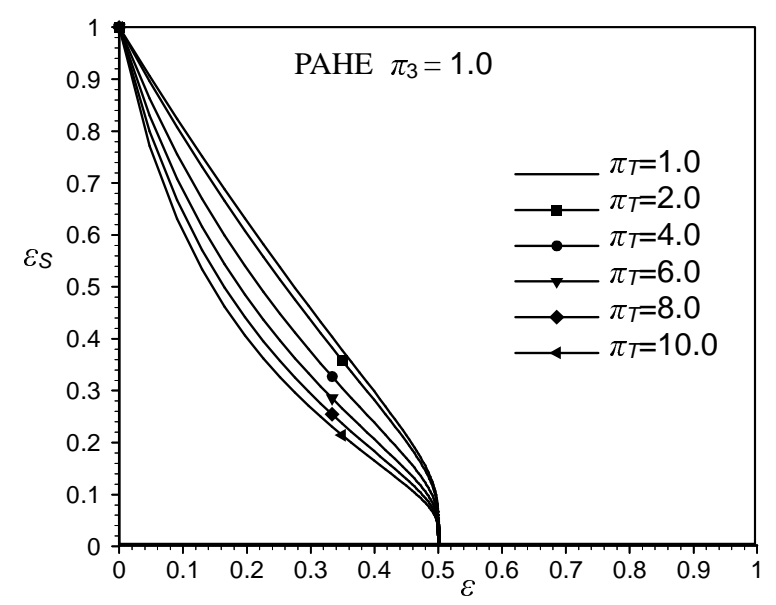

Figure $2 \mathrm{~b}$ Relative entropy generation $\varepsilon_{S}$ as a function of the heat exchanger effectiveness $\varepsilon$ for $\pi_{3}=1.0$ and $\pi_{T}=1$ to 10 , for a parallel flow heat exchanger

Figures $3 \mathrm{a}$ to $4 \mathrm{~b}$ show the relative entropy generation as a function of the heat exchanger effectiveness for the counterflow heat exchanger. Figs. $3 \mathrm{a}$ and $3 \mathrm{~b}$ correspond to $\pi_{3}=0.5$, while Figs. $4 \mathrm{a}$ and $4 \mathrm{~b}$ are for $\pi_{3}=1$. In Figs. $3 \mathrm{a}$ and $4 \mathrm{a}$ the parameter $\pi_{T}$ varies from 0.2 to 1 , and in Figs. $3 \mathrm{~b}$ and $4 \mathrm{~b}$ from 1 to 10. 


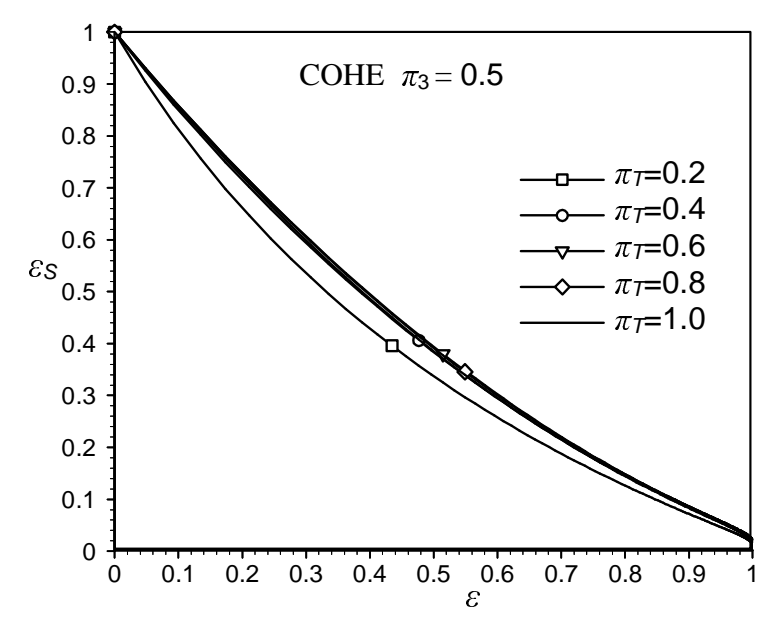

Figure 3a Relative entropy generation $\varepsilon_{S}$ as a function of the heat exchanger effectiveness $\varepsilon$ for $\pi_{3}=0.5$ and $\pi_{T}=0.2$ to 1 , for a counterflow heat exchanger

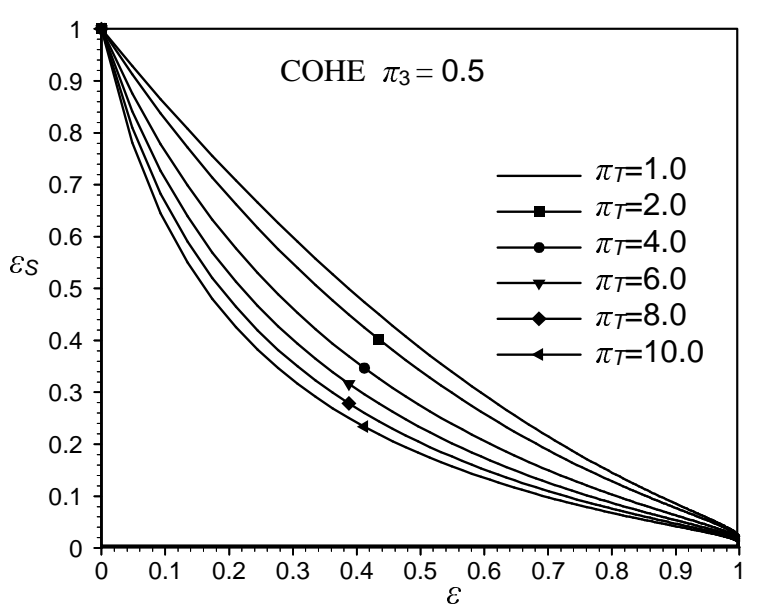

Figure $3 b$ Relative entropy generation $\varepsilon_{S}$ as a function of the heat exchanger effectiveness $\varepsilon$ for $\pi_{3}=0.5$ and $\pi_{T}=1$ to 10 , for a counterflow heat exchanger

From Eq.(19) it follows for a counterflow heat exchanger that for $\pi_{2} \rightarrow \infty, \varepsilon \rightarrow 1.0$, as shown in Figs. $3 \mathrm{a}$ to $4 \mathrm{~b}$.

Figures $5 \mathrm{a}$ to $6 \mathrm{~b}$ show the relative entropy generation as a function of the heat exchanger effectiveness for the cross-flow heat exchanger. Figs. $5 \mathrm{a}$ and $5 \mathrm{~b}$ correspond to $\pi_{3}=0.5$, while Figs. $6 \mathrm{a}$ and $6 \mathrm{~b}$ are for $\pi_{3}=1$. In Figs. 5a and 6a the parameter $\pi_{T}$ varies from 0.2 to 1 and in Figs. $5 \mathrm{~b}$ and $6 \mathrm{~b}$ from 1 to 10. The plotted results were obtained by using a numerical integration [9] giving accurate results at least in three significant digits. 


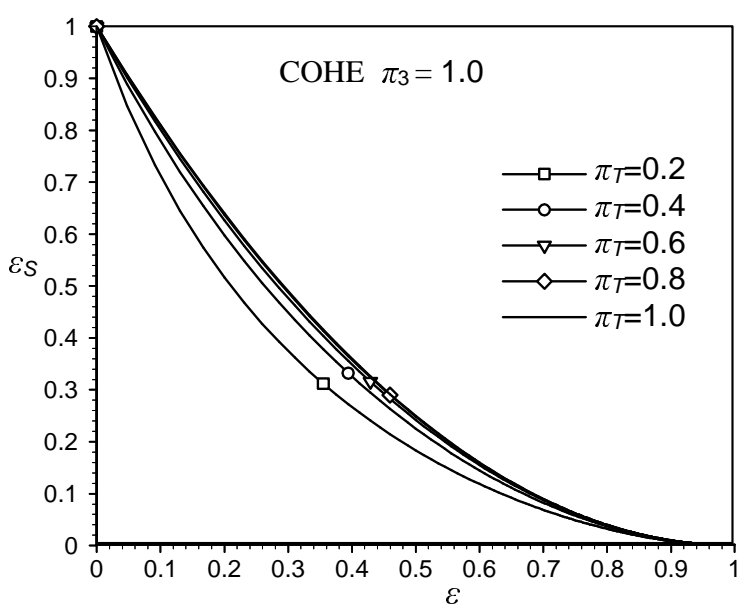

Figure 4a Relative entropy generation $\varepsilon_{S}$ as a function of the heat exchanger effectiveness $\varepsilon$ for $\pi_{3}=1$ and $\pi_{T}=0.2$ to 1 , for a counterflow heat exchanger

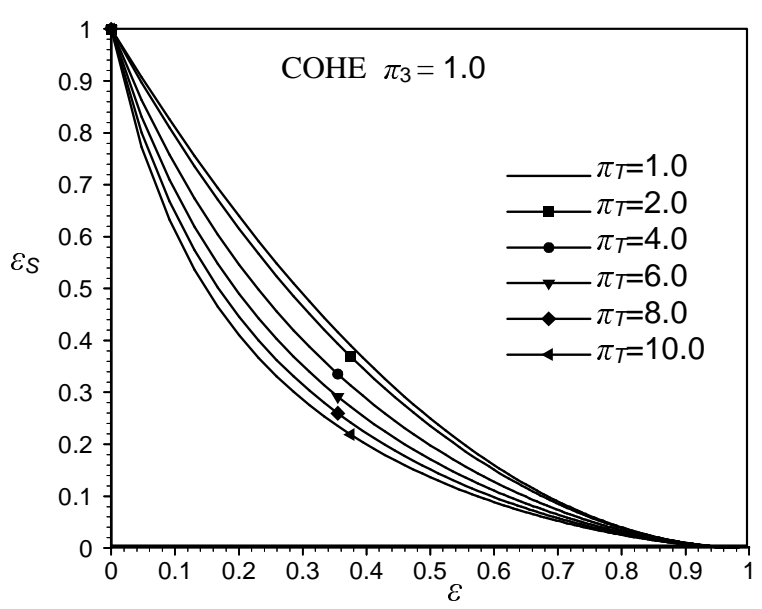

Figure 4b Relative entropy generation $\varepsilon_{S}$ as a function of the heat exchanger effectiveness $\varepsilon$ for $\pi_{3}=1$ and $\pi_{T}=1$ to 10 , for a counterflow heat exchanger 


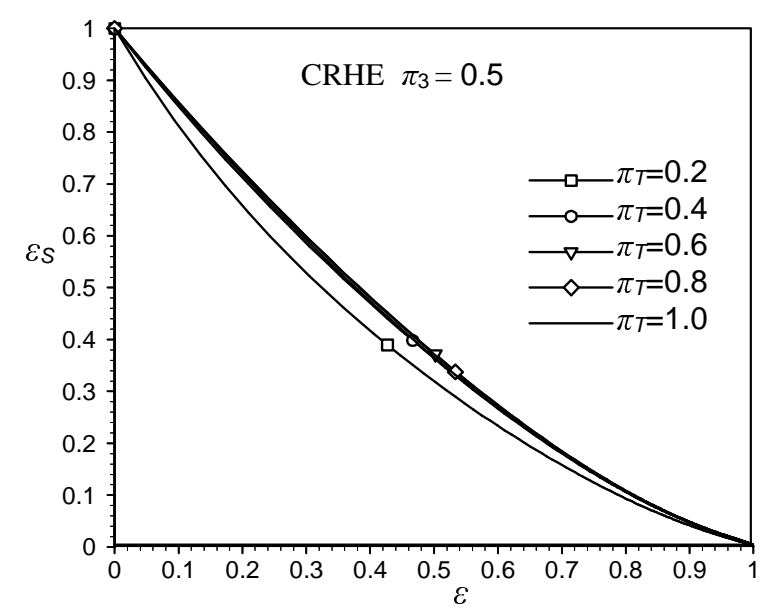

Figure 5a Relative entropy generation $\varepsilon_{S}$ as a function of the heat exchanger effectiveness $\varepsilon$ for $\pi_{3}=0.5$ and $\pi_{T}=0.2$ to 1 , for a cross-flow heat exchanger

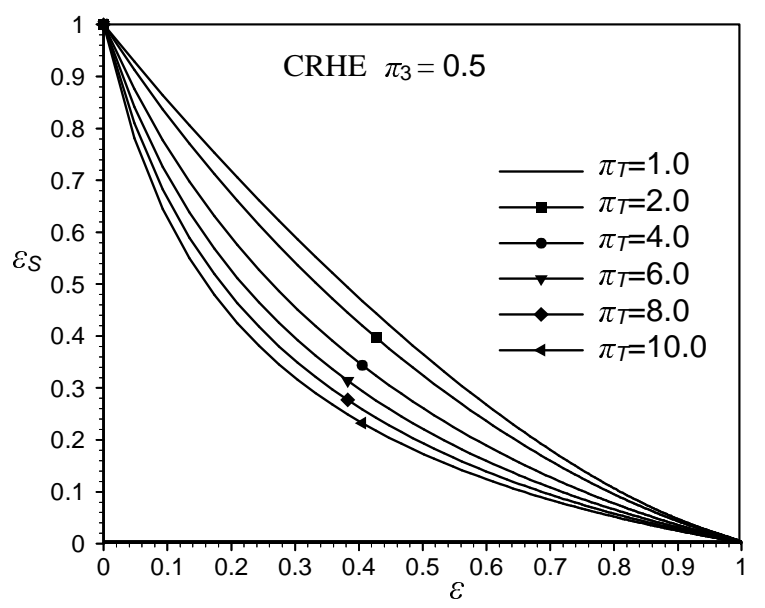

Figure 5b Relative entropy generation $\varepsilon_{S}$ as a function of the heat exchanger effectiveness $\varepsilon$ for $\pi_{3}=0.5$ and $\pi_{T}=1$ to 10 , for a cross-flow heat exchanger 


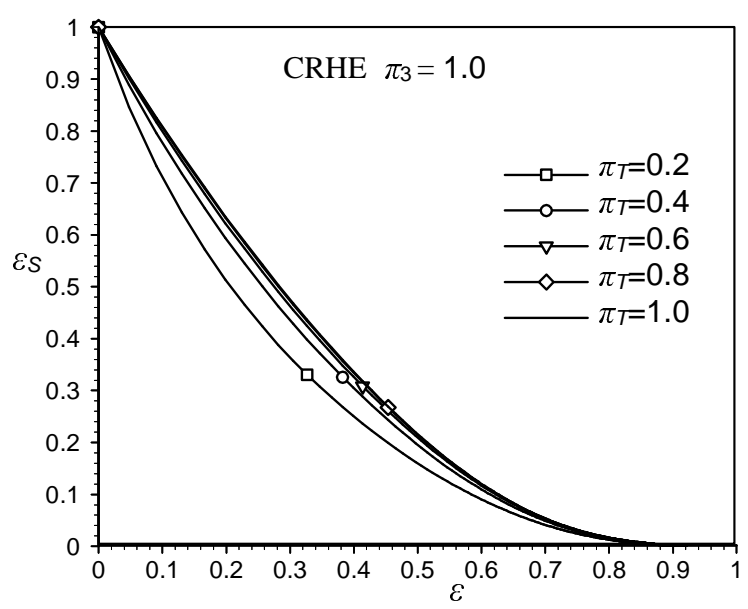

Figure 6a Relative entropy generation $\varepsilon_{S}$ as a function of the heat exchanger effectiveness $\varepsilon$ for $\pi_{3}=1$ and $\pi_{T}=0.2$ to 1 , for a cross flow heat exchanger

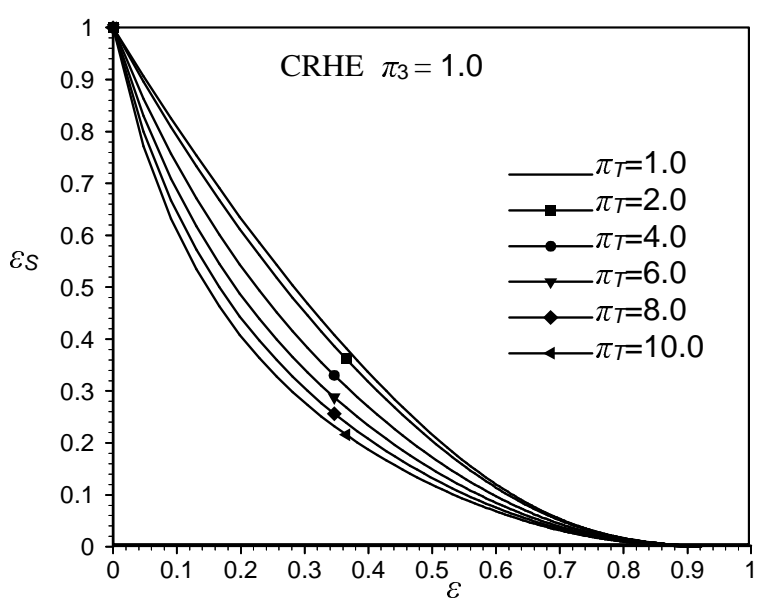

Figure $6 \mathrm{~b}$ Relative entropy generation $\varepsilon_{S}$ as a function of the heat exchanger effectiveness $\varepsilon$ for $\pi_{3}=1$ and $\pi_{T}=1$ to 10 , for a cross-flow heat exchanger

For this type of heat exchanger, the effectiveness also tends to one when $\pi_{2} \rightarrow \infty$, regardless of the parameter $\pi_{T}$. Table 1 shows the values of the relative entropy generation $\varepsilon_{S}$ and parameter $\pi_{2}$ for $\varepsilon=0.4, \pi_{3}=0.5$ and 1.0 and $\pi_{T}=0.2$ and 10 . 
Table 1 Calculated $\varepsilon_{\mathrm{S}}$ and $\pi_{2}$ for $\varepsilon=0.4$ and selected $\pi_{3}$ and $\pi_{\top}$

\begin{tabular}{|c|c|c|c|c|c|}
\hline \multirow{2}{*}{$\begin{array}{c}\text { Heat } \\
\text { exchanger }\end{array}$} & & \multicolumn{4}{|c|}{$\varepsilon=0.4$} \\
\cline { 3 - 6 } & & \multicolumn{2}{|c|}{$\pi_{3}=0.5$} & \multicolumn{2}{c|}{$\pi_{3}=1.0$} \\
\cline { 2 - 6 } & $\pi_{T}=0.2$ & $\pi_{T}=10$ & $\pi_{T}=0.2$ & $\pi_{T}=10$ \\
\hline \multirow{2}{*}{ PAHE } & $\varepsilon_{S}$ & 0.400 & 0.220 & 0.215 & 0.165 \\
& $\pi_{2}$ & 0.610 & 0.610 & 0.805 & 0.805 \\
\hline \multirow{2}{*}{ CRHE } & $\varepsilon_{S}$ & 0.415 & 0.230 & 0.245 & 0.185 \\
& $\pi_{2}$ & 0.590 & 0.590 & 0.710 & 0.710 \\
\hline \multirow{2}{*}{ COHE } & $\varepsilon_{S}$ & 0.420 & 0.235 & 0.265 & 0.195 \\
& $\pi_{2}$ & 0.575 & 0.575 & 0.667 & 0.667 \\
\hline
\end{tabular}

It is obvious from Table 1 that, for the given effectiveness, the counterflow heat exchanger generates most of the entropy rate, but it has the smallest surface area (parameter $\pi_{2}$ ).

\subsection{Cases of an evaporator or a condenser}

In the case of an evaporator or a condenser $\left(C_{2} \rightarrow \infty\right.$ and $\left.\pi_{3}=0\right)$ the entropy generation for all three types of heat exchangers becomes identical, as shown in Eq. (14), so the relative entropy generation can be shown as a function of $\varepsilon$ and $\pi_{T}$, as in Fig. 7 .

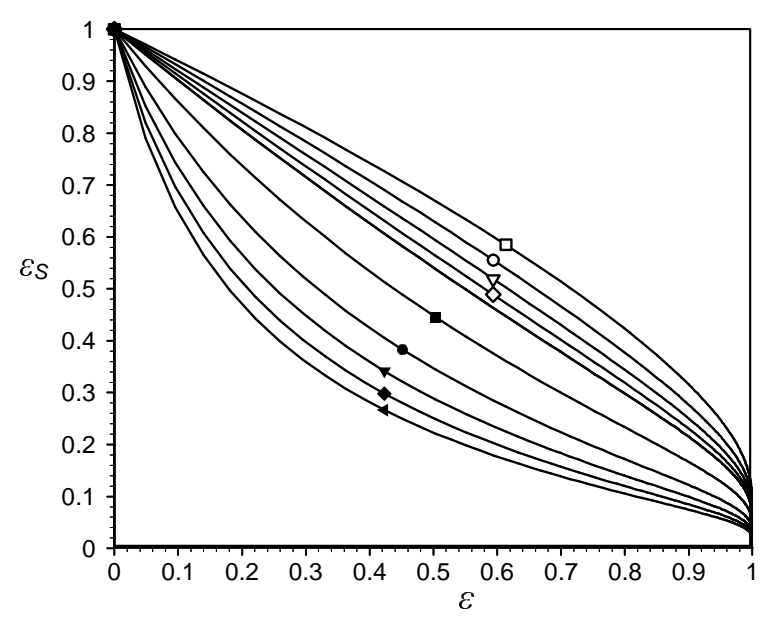

Figure 7 Relative entropy generation $\varepsilon_{S}$ as a function of the heat exchanger effectiveness $\varepsilon$ for $\pi_{T}=0.2$ to 10 and $\pi_{3}=0$ (meaning of symbols is the same as in the previous figures) 
For $\varepsilon \rightarrow 1$, the heat exchanger surface area obviously tends to infinity, and consequently $N_{S} \rightarrow 0$. Since the $N_{S, \max }$ takes a finite value depending on $\pi_{T}$ only, the relative entropy generation tends to zero for $A_{0} \rightarrow \infty$. Further it can be noticed in Fig. 7 that the parametric curves corresponding to $\pi_{T}>1$ are concave, while those for $\pi_{T}<1$ are convex. For the given heat exchanger effectiveness the relative entropy generation grows if drops the $\pi_{T}$. For e.g. $\varepsilon=0.4, \pi_{T}=10$ and $0.2, \varepsilon_{S}$ becomes 0.275 and 0.735 , respectively. For $\varepsilon_{S}=0.5$ and $\pi_{T}=10$ and 0.2 , the achieved heat exchanger effectiveness is 0.18 and 0.715 , respectively.

Fig. 8 shows the relative entropy generation as a function of heat exchanger effectiveness for $\pi_{T}=0.5$ and for three values of $\pi_{3}(0 ; 0.5$ and 1$)$ and for all three types of heat exchangers. The solution for $\pi_{3}=0$ is the same for all the three types.

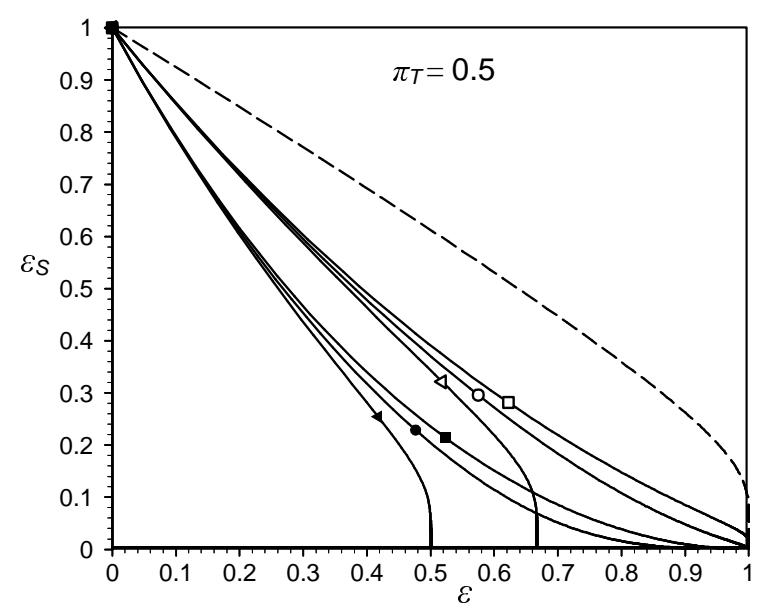

Figure 8 Relative entropy generation $\varepsilon_{S}$ of the PAHE (triangles) COHE (squares) and CRHE (circles) versus the heat exchanger effectiveness $\varepsilon$ for the $\pi_{3}=0$ (dashed line); 0.5 (outlined symbols) and 1 (filled symbols) and for $\pi_{T}=0.5$

It may be seen that in the range $0<\varepsilon<0.2$ and for a given $\pi_{T}$, the type of heat exchanger has no influence on the relative entropy generation. For a given $\varepsilon_{S}$ evaporator or condenser is the most effective, followed by the counterflow, cross-flow and parallel flow heat exchanger respectively. As concluded earlier, for a given $\varepsilon$ the maximal relative entropy generation occurs in an evaporator or condenser, followed by the counterflow heat exchanger, while it becomes minimal in the parallel flow heat exchanger.

\section{Conclusion}

The relative entropy generation is defined as a ratio of actual entropy generation rate and its maximal value. Analytical expressions of relative entropy generation are given for parallel flow and counterflow heat exchangers and analytical-numerical results for cross-flow heat exchangers. An analysis of obtained expressions is carried out for the case of evaporators or condensers. The entropy generation is 
related to the heat exchanger effectiveness, so the heat exchangers can be evaluated quantitatively. Obtained results can be used in a more general procedure for heat exchanger optimization.

\section{REFERENCES}

1. Bošnjaković, F. Kampf den Nichtumkehrbarkeiten; Arch. Wärmewirtsch. Dampfkesselwes. 1938.

2. Bejan, A. Advanced Engineering Thermodynamics; John Wiley \&Sons: New York, 1988.

3. Bejan, A. Entropy Generation Minimization; CRC Press: Boca Ratom, 1996.

4. Hesselgreaves, J. E. Rationalization of second law analysis of heat exchangers. International Journal of Heat and Mass Transfer 2000, 43, 4189-4204.

5. Guo, Z. Y.; Shou, S.; Chen, L. Theoretical analysis and experimental confirmation of the uniformity principle of temperature difference field in heat exchanger. International Journal of Heat and Mass Transfer 2002, 45, 2119-2127.

6. Bošnjaković, F.; Knoche, K. F. Technische Thermodynamik, Teil I, VEB: Leipzig. 1988.

7. Bošnjaković, F.; Knoche, K. F. Technische Thermodynamik, Teil II, Steinkopf: Darmstadt, 1997.

8. Nusselt, W. Forschung auf dem Gebiete der Ingenieur Wesens. Technische Mechanik und Thermodynamik 1930, 1, 417-425.

9. Galović, A.; Živić, M.; Halasz, B. The analysis of the entropy production and heat transfer efficiency of a crossflow heat exchanger, $13^{\text {th }}$ International Conference on Thermal Engineering and Thermogrammetry (THERMO), 18-20 June 2003, Budapest.

\section{NOMENCLATURE}

$A_{0} \quad$ heat exchanger wall surface area, $\mathrm{m}^{2}$

$C$ heat capacity rate, $\mathrm{W} / \mathrm{K}$

$k \quad$ overall heat transfer coefficient, $\mathrm{W} /\left(\mathrm{m}^{2} \cdot \mathrm{K}\right)$

$N_{S} \quad$ entropy generation number

$\dot{S}_{g e n} \quad$ entropy generation rate, W/K

$T$ temperature, $\mathrm{K}$

\section{Greek Letters}

$\Phi \quad$ heat transfer rate, $\mathrm{W}$

$\varepsilon \quad$ heat exchanger effectiveness

$\varepsilon_{S} \quad$ relative entropy generation

$\pi_{2} \quad$ number of transfer units

$\pi_{T} \quad$ ratio of inlet temperatures

$\pi_{3} \quad$ ratio of heat capacity rates

\section{Subscripts}

1 weaker stream

2 stronger stream

max maximum

\section{Superscripts}

inlet

(C) 2003 MDPI (http://www.mdpi.org). Reproduction is permitted for noncommercial purposes. 